\title{
Mitochondrial Dysfunction and Chronic Fatigue Syndromes: Issues in Clinical Care (modified version)
}

\author{
Th. Bihari Singh ${ }^{1}$, Kh.Robindro Singh ${ }^{2}$

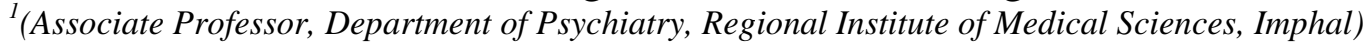 \\ ${ }_{2}^{2}$ (Post-graduate Trainee, Department of Psychiatry, Regional Institute of Medical Sciences, Imphal)
}

\begin{abstract}
Chronic fatigue syndrome (CFS) is an illness characterized by persistent and relapsing fatigue, often accompanied by numerous symptoms involving various body systems. The etiology of CFS remains unclear. Despite considerable worldwide efforts, no single etiology has been identified to explain the development of chronic fatigue syndrome (CFS). It is likely that multiple factors promote its development, sometimes with the same factors both causing and being caused by the syndrome. However, a number of recent studies have shown oxidative stress may be involved in its pathogenesis. Any dysfunction in the Mitochondria, the key organelle responsible for cellular energy production can result in the excess fatigue and other symptoms that are common complaints in almost every chronic disease. Several components of this system require routine replacement, and this need can be facilitated with natural supplements. A detailed review of the literature suggests a number of marginal nutritional deficiencies may have etiologic relevance. In this review article an attempt has been made to look into these factors in relation to chronic fatigue syndrome.
\end{abstract}

Keywords: fatigue, mitochondria, ATP, energy, apoptosis, oxidative stress

\section{Introduction}

Chronic fatigue syndrome (CFS) is a relatively common disorder, particularly in women, affecting 522 women and 291 men per $100,000 .{ }^{1}$ In addition to the characteristic persistent fatigue, CFS patients often complain of a number of symptoms including headache, joint pain, gastrointestinal (GI) disturbance, cognitive dysfunction, visual disturbance, and paresthesia. ${ }^{2,3}$ Despite considerable worldwide efforts, no single etiology has been found to explain the syndrome. It is likely that multiple factors promote its development, sometimes with the same factors both causing and being caused by the syndrome. Many of these factors constitute specific pathophysiological entities that characterize certain subsets of chronic fatigue patients. Numerous factors appear to promote the development of the syndrome. ${ }^{4}$

\section{Mitochondria Structure And Function}

The largest number of mitochondria is found in the most metabolically active cells, such as skeletal and cardiac muscle and the liver and brain. Mitochondria are found in every human cell except mature erythrocytes (Cohen and Gold, 2001). Mitochondria produce more than $90 \%$ of our cellular energy by OXPHOS (Chance et al., 1979). Energy production is the result of two closely coordinated metabolic processes - the tricarboxylic acid (TCA) cycle, also known as the Kreb's or citric acid cycle, and the electron transport chain (ETC). The TCA cycle converts carbohydrates and fats into some ATP, but its major job is producing the coenzymes NADH and FADH so that they, too, are entered into the ETC. ${ }^{5}$

\section{Mitochondrial Dysfunction And Chronic Fatigue Syndrome}

Mitochondrial dysfunction is directly related to excess fatigue. Fatigue is considered a multidimensional sensation that is perceived to be a loss of overall energy and an inability to perform even simple tasks without exertion. Although mild fatigue can be caused by a number of conditions, including depression and other psychological conditions, moderate to severe fatigue involves cellular energy systems. At the cellular level, moderate to severe fatigue is related to loss of mitochondrial function and diminished production of ATP. ${ }^{6}$

Suspected aetiologies for Chronic Fatigue Syndrome are viral infections and the post viral fatigue syndrome, fibromyalgia, neurally-mediated hypotension, psychogenic biological dysfunction, low natural killer cell syndrome. Factors suspected of promoting Chronic Fatigue Syndrome include hypoxemia endocrine dysfunction, immune dysfunction, stress-related dysfunction, somatoform disorder, marginal nutritional deficiencies, intestinal hyperpermeability, overgrowth of pathogenic intestinal flora (dysbiosis), food and chemical sensivities, chemical toxicity, heavy metal toxicity. ${ }^{3,7,8,9,10}$ 


\section{Mitochondrial Dysfunction And Depression}

Adjusting to a chronic, debilitating illness can sometimes lead to depression. As many as half of CFS patients develop depression at some time during the course of their illness. When it is present, depression needs to be treated. Although treating depression can reduce anxiety and stress, it is not a cure for CFS. New theories about the pathophysiology of depression and the action of antidepressant treatment proposes that mood disorders are caused by structural or functional changes in particular molecules and signalling pathways in the brain, and that antidepressants function by counteracting these molecular changes. It is supposed that structural and functional brain abnormalities in patients with depressive disorder may be associated with low levels of brain-derived neurotrophic factor (BDNF), abnormal function of hypothalamic pituitary adrenal (HPA) axis, glutamatergic toxicity, activation of inflammatory and cell-mediated immune response, decreased antioxidant capacity and increased oxidative and nitrosative stress, disturbed chronobiological rythms, and mitochondrial dysfunctions.

\section{Mitochondrial Dysfunction In Ageing}

The role of accumulating mitochondrial defects as a cause of ageing remains highly controversial. There is widespread belief that mitochondrial-generated oxidative stress and accumulated gene defects may itself cause ageing. A recent TV documentary likened accumulated oxidative stress in ageing to the oxidation involved in a rusting motor car. ${ }^{11,12}$

It also encourages the idea that ageing is associated with accumulating oxidative damage. ${ }^{13}$

\section{Treatment Approach With Natural Supplements}

\section{Coenzyme Q10 (ubiquinone)}

Coenzyme Q10 (CoQ10 [ubiquinone]) is a key cofactor and component of the mitochondrial electron transport chain and one of the most widely used natural supplements. ${ }^{14,15}$ CoQ10 is found in all cell and organelle membranes, where it can participate in redox shuttling. It has an important intracellular signalling role, as well as both antioxidant and pro-oxidant roles. CoQ10 modulates the mitochondrial permeability transition pore involved in apoptosis and activates uncoupling proteins. Because CoQ10 is an important component of the mitochondrial oxidative phosphorylation system, its supplementation in individuals with reduced levels should result in increased energy production and reduced fatigue. In plasma, CoQ is largely (95\%) bound to lipoproteins in the reduced ubiquinol form..$^{15,16}$

Standard dosage: Ubiquinol doses of 2 to $8 \mathrm{mg} / \mathrm{kg}$ per day (administered twice daily with meals) seem prudent; this form of CoQ10 in a solubilised, bioavailable form is preferred over ubiquinone. Ubiquinone doses of 5 to $30 \mathrm{mg} / \mathrm{kg}$ per day (administered in two divided doses daily with meals) is an available alternative. ${ }^{17}$

\section{L-Carnitine}

L-carnitine (3-hydroxy-4-N-trimethylaminobutyrate) is a naturally occurring fatty acid transporter found in all species of mammals. L- Carnitine is a cellular compound that plays a critical role in the process of mitochondrial beta-oxidation of fatty acids and the esterifcation of free fatty acids that may otherwise be sequestered by CoA. Carnitine transfers long-chain fatty acids across the mitochondrial inner membrane as acylcarnitine esters. These esters are oxidized to acetyl CoA, which enters the Krebs cycle and results in subsequent generation of ATP via oxidative phosphorylation. Certain tissues, such as skeletal muscle, heart, and liver, largely depend on beta-oxidation for ATP production. Carnitine may prevent CoA depletion and remove excess, potentially toxic, acyl compounds; at this time there is no pharmacologic method to increase CoA levels. L-carnitine supplementation has been successfully used in clinical disorders that are characterized by low concentrations of L-carnitine or impaired fatty acid oxidation, such as diabetes, sepsis, renal disease, and cardiomyopathy. Diet is the source of $75 \%$ of carnitine; $25 \%$ is synthesized in the body, predominantly within muscle, liver, and kidneys ${ }^{18}$.

\section{Standard dosage:}

Oral: Children: $20-100 \mathrm{mg} / \mathrm{kg} / \mathrm{day}$ divided into two or three doses; higher doses have been used. Adults: $330-990 \mathrm{mg} / \mathrm{dose}$ two or three times per day; $3 \mathrm{~g} / \mathrm{day}$ is the usual maximum. IV: Children and adults: $50-100 \mathrm{mg} / \mathrm{kg} /$ day divided every $4-6$ hours, increased as needed to a maximum of $300 \mathrm{mg} / \mathrm{kg} / \mathrm{day}$.

\section{L-Creatine}

Creatine, a compound present in cells, combines with phosphate in the mitochondria to form phosphocreatine. It serves as a source of high-energy phosphate, released during anaerobic metabolism. It also acts as an intracellular buffer for ATP and as an energy shuttle for the movement of high-energy phosphates from mitochondrial sites of production to cytoplasmic sites of utilization. The highest concentrations of creatine 
are found in tissues with high energy demands, such as skeletal muscle and brain. Creatine is continuously replaced through a combination of diet and endogenous synthesis ${ }^{19}$.

Standard dosage Adult: $10 \mathrm{~g} /$ day, divided into two doses. Pediatric: $0.1 \mathrm{~g} / \mathrm{kg}$ per day, divided into two doses.

\section{L-Arginine}

Arginine is a semi-essential amino acid involved in growth, urea detoxifcation, and creatine synthesis. L-arginine produces nitric oxide, which has neurotransmitter and vasodilatory properties. An initial small study demonstrated that intravenous (IV) administration of L-arginine $(500 \mathrm{mg} / \mathrm{kg} / \mathrm{dose})$ quickly decreased the severity of strokelike symptoms, enhanced the dynamics of microcirculation, and reduced tissue injury from ischemia in patients with mitochondrial encephalomyopathy, lactic acidosis, and strokelike episodes $(\text { MELAS })^{20}$.

Standard dosage Acute stroke: $500 \mathrm{mg} / \mathrm{kg}$ IV per day for 1-3 days. Maintenance dose: $150-300 \mathrm{mg} / \mathrm{kg}$ orally or IV daily, divided into two or three doses.

\section{NADH}

In neurodegenerative diseases, oxidative damage is extensive, and various mitochondrial antioxidants have been used to treat disease and delay progression. NADH functions as a cellular redox cofactor in over 200 cellular redox reactions and as substrate for certain enzymes. Humans universally require NADH, and its deficiency results in pellagra, which is characterized by dermatitis, diarrhoea, dementia, and eventually death. In the mitochondria, NADH delivers electrons from metabolite hydrolysis to the electron transport chain, but in its reduced form, it can also act as a strong antioxidant. The usual route of dietary supplementation has historically been via NADH precursors, such as niacin, nicotinic acid, or nicotinamide, but recently, microcarriers have been used to stabilize oral NADH so that it can be directly ingested in small doses and absorbed in the gastrointestinal system. This supplementation turns out to be more effective than large oral doses of NADH, as in some studies that used up to $50 \mathrm{mg} / \mathrm{kg} / \mathrm{day} .^{21}$

\section{Alpha-Lipoic Acid}

Alpha-lipoic acid (a-lipoic acid [1,2-dithiolane-3-penta-noic acid]) is a potent antioxidant, transition metal-ion chelator, redox transcription regulator, and anti-inflammatory agent. It acts as a critical cofactor in mitochondrial, alpha-keto acid dehydrogenases, and thus, it is important in mitochondrial, oxidativedecarboxylation reactions. Clinically, a-lipoic acid has been used as an oral supplement in the treatment of complications associated with diabetes mellitus, and according to a review by Shay et al. As a result of aging and in many chronic diseases, certain sphingolipids especially ceramides, and in particular, shortchain ceramides accumulate in mitochondria due to hydrolysis of sphingomyelin by sphingomyelinase, and eventually, this accumulation retards electron transport activity. Ceramide accumulation in mitochondria is especially damaging to cardiac tissue. As mentioned above, in diabetes a-lipoic acid has been used extensively to reduce diabetic complications, such as sensorimotor polyneuropathies. ${ }^{22}$

\section{Membrane Phospholipids}

The dietary replacement of mitochondrial membrane phospholipids (lipid replacement therapy [LRT]) using food derived molecules to remove damaged, mainly oxidized, membrane lipids in mitochondria and other cellular organelles has proved very effective at increasing mitochondrial function and reducing fatigue. To some degree, antioxidant supplements can reduce ROS/RNS levels and prevent some oxidation of mitochondrial membrane phospholipids, but antioxidants alone cannot repair the damage already done to cells, and in particular, to cells' mitochondrial inner membranes. ${ }^{23}$

The use of oral membrane phospholipids plus antioxidants in doses ranging from 500 to $2000 \mathrm{mg}$ per day has been effective in the treatment of certain clinical conditions, such as chronic fatigue and Fatiguing illnesses. LRT results in the actual replacement of damaged membrane phospholipids with undamaged (unoxidized) lipids to ensure proper function of cellular and especially mitochondrial membranes. Oral membrane phospholipids can increase mitochondrial function and decrease fatigue in chronic fatigue syndrome, fibromyalgia syndrome, and other fatiguing conditions, including natural aging. ${ }^{24}$

\section{Conclusion}

As deficiencies of various B vitamins, vitamin C, magnesium, sodium, zinc, L-tryptophan, L-carnitine, coenzyme Q10, and essential fatty acids may have etiologic relevance and in fact clinical trials have also shown the utility of using oral replacement supplements, such as L-carnitine, alpha-lipoic acid (a-lipoic acid [1,2dithiolane-3-pentanoic acid]), coenzyme Q10 (CoQ10 [ubiquinone]), NADH (reduced nicotinamide adenine dinucleotide), membrane phospholipids, and other supplements. Combinations of these supplements can reduce 
significantly the fatigue and other symptoms associated with chronic disease and can naturally restore mitochondrial function, even in long-term patients with intractable fatigue.

\section{References}

[1]. Jason LA, Richman JA, Rademaker AW, et al. A community-based study of chronic fatigue syndrome. Arch Intern Med 1999;159:2129-2137.

[2]. Komaroff AL, Buchwald D. Symptoms and signs of chronic fatigue syndrome. Rev Infect Dis 1991;13:S8 S11.

[3]. Komaroff AL, Fagioli LR, Geiger AM, et al. An examination of the working case definition of chronic fatigue syndrome. Am J Med 1996;100:56-64

[4]. Melvyn R. Werbach. Nutritional Strategies for Treating Chronic Fatigue Syndrome. Alternative Medicine Review Volume 5, Number $2 \diamond 2000$

[5]. S.R. Pieczenik, J. Neustadt. Mitochondrial dysfunction and molecular pathways of disease / Experimental and Molecular Pathology 83 (2007) 84-92

[6]. Rich PR, Marechal A. The mitochondrial respiratory chain. Essays Biochem. 2010;47:1-23.

[7]. Nicholls DG. Mitochondrial ion circuits. Essays Biochem. 2010;47:25-35.

[8]. Kroenke K, Wood DR, Mangelsdorff AD, Meier NJ, Powell JB. Chronic fatigue in primary care: prevalence, patient characteristics, and outcome. JAMA. 1988;260(7):929-934.

[9]. Nicolson GL, Settineri R. Lipid Replacement Therapy: a functional food approach with new formulations for reducing cellular oxidative damage, cancerassociated fatigue and the adverse effects of cancer therapy. Funct Foods Health Dis. 2011;1(4):135-160.

[10]. Morrison JD. Fatigue as a presenting complaint in family practice. J Family Pract.1980;10(5):795-801.

[11]. Trifunovic, A., Wredenberg et al (2004) Premature ageing in mice expressing defective mitochondrial DNA polymerase. Nature 429, 417-423

[12]. Kujoth, G.C. et al (2005) Mitochondrial DNA mutations, oxidative stress, and apoptosis in mammalian ageing. Science 309, 481484

[13]. Migliaccio. E et al (1999) The p66shc adaptor protein controls oxidative stress response and life span in mammals. Nature 402, 309313

[14]. Kerr DS. Treatment of mitochondrial electron transport chain disorders: a review of clinical trials over the past decade. Mol Genet Metab. 2010;99(3):246255

[15]. Littarru GP, Tiano L. Clinical aspects of coenzyme Q10: an update. Nutrition. 2010;26(3):250-254

[16]. Orsucci D, Mancuso M, Ienco EC, LoGerfo A, Siciliano G. Targeting mitochondrial dysfunction and neurodegeneration by means of coenzyme Q10 and its analogues. Curr Med Chem. 2011;18(26):4053-4064.

[17]. Parikh et al. A modern approach to the treatment of Mitochondrial diseases. Current Treatment Options in Neurology 2009, 11:414-430

[18]. Tein I: Carnitine transport: pathophysiology and metabolism of known molecular defects. J Inherit Metab Dis 2003, 26:147-169.

[19]. Borchert A, Wilichowski E, Hanefeld F: Supplementation with creatine monohydrate in children with mitochondrial encephalomyopathies. Muscle Nerve 1999, 22:1299-1300.

[20]. Koga Y, Ishibashi M, Ueki I, et al.: Effects of L-arginine on the acute phase of strokes in three patients with MELAS. Neurology 2002, 58:827-828.

[21]. Birkmayer JG. Coenzyme nicotinamide adenine dinucleotide: new therapeutic approach for improving dementia of the Alzheimer type. Ann Clin Lab Sci.1996;26(1):1-9.

[22]. Ziegler D, Nowak H, Kempler P, Vargha P, Low PA. Treatment of symptomatic diabetic polyneuropathy with the antioxidant alpha-lipoic acid: a meta-analysis. Diabet Med. 2004;21(2):114-121

[23]. Nicolson GL. Lipid replacement as an adjunct to therapy for chronic fatigue, anti-aging and restoration of mitochondrial function. $\mathbf{J}$ Am Nutraceutical Assoc. 2003;6(3):22-28.

[24]. Ellithorpe RR, Settineri RA, Nicolson GL. Pilot study: reduction of fatigue by use of a dietary supplement containing glycophospholipids. J Am Nutraceutical Assoc. 2003;6(1):23-28. 